\title{
Crystal structure of the p38a MAP kinase in complex with a docking peptide from TAB1
}

\author{
XIN FengJiao \& WU JiaWei* \\ Tsinghua-Peking Center for Life Sciences and MOE Key Laboratory of Protein Science, School of Life Sciences, Tsinghua University, Beijing \\ 100084, China
}

Received April 21, 2013; accepted May 9, 2013; published online May 29, 2013

\begin{abstract}
The mitogen-activated protein kinase (MAPK) p38 $\alpha$ is a key regulator in many cellular processes, whose activity is tightly regulated by upstream kinases, phosphatases and other regulators. Transforming growth factor- $\beta$ activated kinase 1 (TAK1) is an upstream kinase in $\mathrm{p} 38 \alpha$ signaling, and its full activation requires a specific activator, the TAK1-binding protein (TAB1).

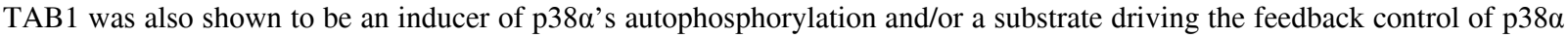
signaling. Here we determined the complex structure of the unphosphorylated p38 $\alpha$ and a docking peptide of TAB1, which shows that the TAB1 peptide binds to the classical MAPK docking groove and induces long-range conformational changes on $\mathrm{p} 38 \alpha$. Our structural and biochemical analyses suggest that TAB1 is a reasonable substrate of p38 $\alpha$, yet the interaction between the docking peptide and $\mathrm{p} 38 \alpha$ may not be sufficient to trigger trans-autophosphorylation of p $38 \alpha$.
\end{abstract}

p38 $\alpha$ MAP kinase, TAB1, KIM, crystal structure

Citation: Xin F J, Wu J W. Crystal structure of the p38 $\alpha$ MAP kinase in complex with a docking peptide from TAB1. Sci China Life Sci, 2013, 56: 653-660, doi: 10.1007/s11427-013-4494-0

The mitogen-activated protein kinase (MAP kinase, MAPK) signaling plays important roles in various cellular processes [1]. A conventional MAPK pathway is composed of three tiers of sequentially activated kinases, termed MAPK kinase kinase (MKKK), MAPK kinase (MKK) and MAPK. In the canonical MAPK pathway, the upstream MAPK kinase kinase phosphorylates and activates the MAPK kinase, which in turn phosphorylates and activates the downstream MAPK. The p38 kinases are a subgroup of MAPK family, comprising of four isoforms, $\alpha, \beta, \gamma$ and $\delta$. These serine/threonine kinases are highly conserved and involved in inflammatory responses, differentiation, tumor suppression, cell cycle and apoptosis [2,3]. Two members of MKK family, MKK3 and MKK6, have high activity toward p38 MAPKs. MKK3 appears to favor the $\mathrm{p} 38 \alpha$ and $\mathrm{p} 38 \beta$ isoforms, while MKK6 phosphorylates all p38 family members. Both MKK3 and
MKK6 are dual-specificity kinases capable of phosphorylating two adjacent Thr and Tyr residues (the Thr-Gly-Tyr motif) in the activation loop of p38 MAP kinases [4]. Upon dual phosphorylation, the p38 MAPKs become active and consequently phosphorylate a large set of substrates such as transcription factors, tumor suppressors and other kinases.

Although the canonical cascade is considered to be the principal activation mechanism of MAPK, three alternative mechanisms have been reported for $\mathrm{p} 38 \alpha$. One involves the direct interaction of $\mathrm{p} 38 \alpha$ with TGF- $\beta$-activated protein kinase 1 binding protein 1 (TAB1), which results in the autophosphorylation and activation of $\mathrm{p} 38 \alpha$ [5]. The second MKK-independent pathway, exclusively in T-cells, involves phosphorylation of Tyr323 on p38 $\alpha$ by the ZAP-70 tyrosine kinase, which leads to $\mathrm{p} 38 \alpha$ autophosphorylation solely on Thr180 of the Thr-Gly-Tyr motif and thus activates p38 $\alpha$ [6,7]. In addition, evidence indicates that phosphatidylino-

*Corresponding author (email: jiaweiwu@mail.tsinghua.edu.cn) 
sitol ether lipid analogues can activate $\mathrm{p} 38 \alpha$ via MKK independent and dependent mechanisms $[8,9]$.

The TGF- $\beta$-activated protein kinase 1 (TAK1) is a MKKK in p38 signaling pathway and plays an important role in the regulation of p38 activity by phosphorylating and activating MKK3 and MKK6 [10]. TAB1 was originally identified as a key regulator of TAK1 that binds directly to the kinase domain of TAK1 and promotes autophosphorylation of two threonine residues (Thr184 and Thr187) and one serine residue (Ser192) within the activation loop of TAK1 kinase [11-13]. Ge et al. [5] later found that TAB1 can interact with p38 $\alpha$ but no other p38 family members, and the interaction of TAB1 with $\mathrm{p} 38 \alpha$ leads to dual autophosphorylation of $\mathrm{p} 38 \alpha$ on both Thr and Tyr sites, in vitro and in vivo. The TAB1-dependent $\mathrm{p} 38 \alpha$ activation appears to play important roles in some physiological and pathological processes, such as injury during myocardial ischemia, maturation of monocyte- derived dendritic cells, maintenance of peripheral T-cell anergy, and intracellular infection of parasite induced interleukin-12 production [1,14-17]. On the other hand, Cheung et al. [18] suggested that the activated p38 $\alpha$ can phosphorylate TAB1, which feedback controlled the activation of TAK1 and reduced the sequential activation of MKK3/6 and p38 $\alpha$. To understand the molecular basis for TAB1- enhanced p38 $\alpha$ autophosphorylation, we solved the crystal structure of $\mathrm{p} 38 \alpha$-TAB 1 peptide complex at $2.7 \AA$ resolution, with the classical hydrophobic docking groove on $\mathrm{p} 38 \alpha$ accommodating the TAB1 peptide via both hydrophobic and hydrophilic interactions. The TAB1induced conformational changes of p38 $\alpha$ and further biochemical analyses elucidate the role of the p38 $\alpha$-TAB 1 interaction.

\section{Materials and methods}

\subsection{Cloning, expression, and purification}

The cDNAs encoding mouse p38 $\alpha$ and human MKK6CA (207E/T211E) were generous gifts from Dr. Wu ZhenGuo, the cDNA of human TAB1 was kindly provided by Dr. Han JiaHuai, and the plasmid of pGEX-4T-1-ATF2 (1-109) was a generous gift from Dr. Choi Eui-Ju. The p38 $\alpha$ and MKK6CA were subcloned into pETDuet-1 (Novagen), and overexpressed in BL21 (DE3) cells. The N-terminal His $_{6}$-tagged bisphosphorylated $\mathrm{p} 38 \alpha(\mathrm{p} 38 \alpha / p \mathrm{~T} p \mathrm{Y})$ was purified through Ni-NTA column (Qiagen) and ion exchange column (Source 15Q, GE Healthcare). The site-specific mutations of TAB1 were generated by standard quick change PCR procedure and verified by sequencing. All TAB1 constructs were subcloned into pET21b (Novagen), and purified as aforementioned. The GST-tagged ATF2 (1-109) was purified via GST affinity column (GE Healthcare) and Source 15Q anion exchange chromatography. The construct of p38 $\alpha$-pepTAB1 fusion protein was generated by overlap PCR procedure, and subcloned into vector $\mathrm{pET} 21 \mathrm{~b}$. The fusion protein for crystallization was purified through Ni-NTA and Source 15Q columns and further polished by size exclusion chromatography (Superdex 200, GE Healthcare). The final concentration was up to $15 \mathrm{mg} \mathrm{mL}{ }^{-1}$ and stored in buffer containing $10 \mathrm{mmol} \mathrm{L}^{-1}$ Hepes pH 7.4, $150 \mathrm{mmol} \mathrm{L}^{-1} \mathrm{NaCl}$ and $2 \mathrm{mmol} \mathrm{L}^{-1}$ DTT. Protein stocks for kinetic analyses were supplemented with glycerol at a final concentration of $20 \%(\mathrm{v} / \mathrm{v})$.

\subsection{Kinetic analyses of p38a}

The autophosphorylation of $\mathrm{p} 38 \alpha$ was carried out at $25^{\circ} \mathrm{C}$ in reaction buffer containing $50 \mathrm{mmol} \mathrm{L}{ }^{-1}$ Hepes pH 7.4, 20 mmol L ${ }^{-1} \mathrm{MgCl}_{2}$, while the TAB1-induced p38 $\alpha$ autophosphorylation was performed in the same buffer supplemented with equal molar of wild type TAB1. At indicated time intervals, aliquots were removed from the reservoir. After $4 \mathrm{~h}$, some $200 \mathrm{nmol} \mathrm{L}^{-1}$ of MKK6CA was introduced into the reaction system, and samples were taken at 10 and $30 \mathrm{~min}$ intervals. All samples were then analyzed by Western blotting using phosphor-Thr (catalog number 9381S, Cell Signaling Technology, Inc.) and phosphor-Tyr antibodies (catalog number 9411, Cell Signaling Technology, Inc.). TAB1 or ATF2 $\left(5 \mu \mathrm{mol} \mathrm{L}{ }^{-1}\right)$ was phosphorylated by dual phosphorylated $\mathrm{p} 38 \alpha / p \mathrm{~T} p \mathrm{Y}$. At indicated time intervals, aliquots were removed from the reservoir and then analyzed by Western blotting using phosphor-Thr antibody.

\subsection{Crystallization and structure determination}

Crystals of $\mathrm{p} 38 \alpha$-pepTAB1 fusion protein were grown using the hanging-drop vapor diffusion method at room temperature by mixing the protein with an equal volume of reservoir solution containing $100 \mathrm{mmol} \mathrm{L}^{-1}$ Hepes, $\mathrm{pH} 7.86$ and $22 \%$ polyacrylic acid 5100 . Fresh crystals were quickly transferred to the cryo-protectant buffer containing reservoir solution supplemented with $25 \%$ (v/v) Ethylene Glycol and then flash-frozen under a cold nitrogen stream at $100 \mathrm{~K}$. The diffraction data was collected at Shanghai Synchrotron Radiation Facility beamline 17U with a Mar225 CCD detector, and processed by HKL2000 [19]. The structure was solved by molecular replacement using Phaser [20] with the p38 $\alpha$-pepMKK3b complex (PDB ID: 1LEZ) as search model, and then refined with PHENIX [21] and Coot [22]. PROCHECK [23] was used to analyze the model stereochemistry. All structural representations were prepared with PyMOL (http://www.pymol.org).

\section{Results and discussion}

\subsection{Biochemical properties of TAB1}

We purified the full-length TAB1 and $\mathrm{p} 38 \alpha$ proteins and 
examined their interaction by gel filtration assay. When eight micromolar equivalents of $\mathrm{p} 38 \alpha$ were mixed with three micromolar equivalents of full-length TAB1, a small amount of p38 $\alpha$ was shifted to earlier fractions and co-migrates with TAB1, while the excess amount of p38 $\alpha$ was eluted as a monomer (Figure 1A). This result clearly indicates that TAB 1 and $\mathrm{p} 38 \alpha$ can form a binary complex in solution. To test the biochemical consequences of the TAB1-p38 $\alpha$ complex, we first performed in vitro kinase assays using the dual phosphorylation of Thr180 and Tyr182 in the activation loop of $\mathrm{p} 38 \alpha$ as the readout of $\mathrm{p} 38 \alpha$ autophosphorylation. As shown in Figure 1B, p38 $\alpha$ was incapable of undergoing autophosphorylation, whereas the presence of TAB1 triggered the autophosphorylation of p38 $\alpha$. However, the TAB1-induced phosphorylation requires hours to achieve significant stoichiometry (2\%-5\%), suggesting that the p38 $\alpha$ autophosphorylation is unlikely to contribute significantly to the in vitro activation of $\mathrm{p} 38 \alpha$. On the other hand, this $\mathrm{p} 38 \alpha$ preparation used in our experiments could be rapidly phosphorylated by its upstream kinase MKK6CA (Figure 1B). We also assessed kinase activity of the dual phosphorylated $\mathrm{p} 38 \alpha / p \mathrm{~T} p \mathrm{Y}$ toward ATF2 and TAB1 (Figure 1C). As reported, ATF2 was efficiently phosphorylated by the activated $\mathrm{p} 38 \alpha / p \mathrm{~T} p \mathrm{Y}$ and approached a plateau in approximately $2 \mathrm{~h}$. In comparison, although TAB 1 can be phosphorylated by $\mathrm{p} 38 \alpha$, the phosphorylation rate of TAB1 was approximately 10 -fold slower than that of p38 $\alpha$-catalyzed ATF2 phosphorylation. These data demonstrate that TAB1 used in our experiments can interact with p38 $\alpha$ directly and is a good substrate for p38 $\alpha$ MAP kinase. Since TAB1 is an essential activator of the p38 $\alpha$ upstream kinase TAK1 [10] and the phosphorylation sites on TAB1 lie in the proximity of its TAK1-interacting motif [18], it comes naturally that the TAB1 phosphorylation by $\mathrm{p} 38 \alpha$
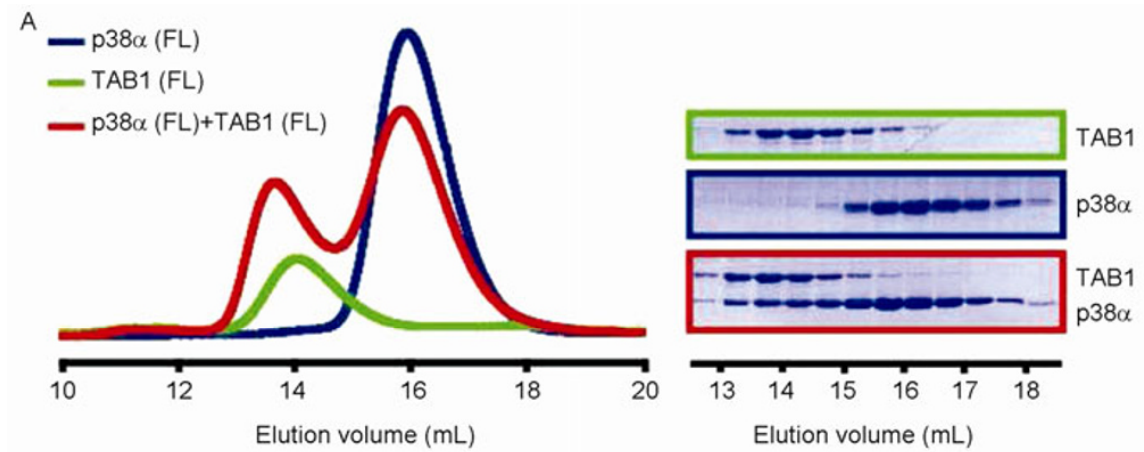

+ MKK6CA

$\left(200 \mathrm{nmol} \mathrm{L}^{-1}\right)$

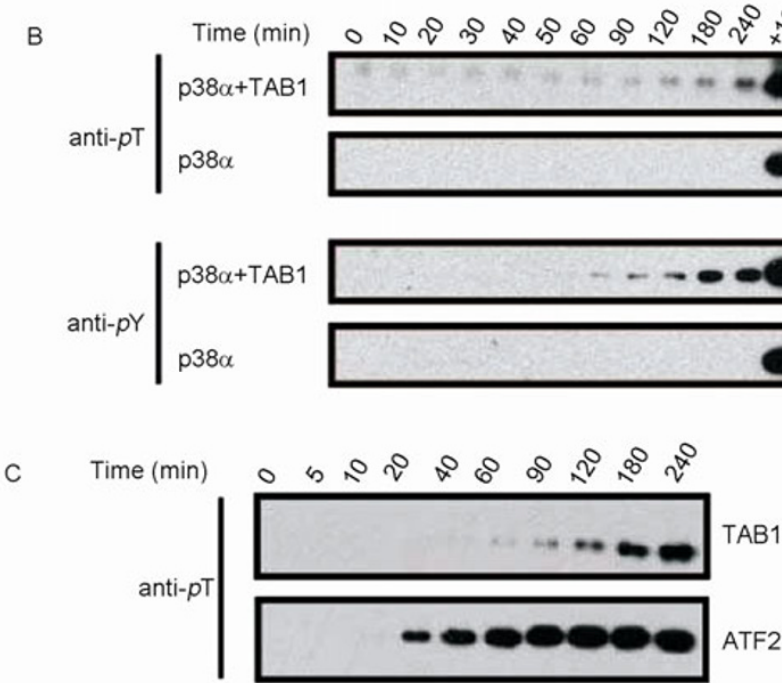

Figure 1 Biochemical studies of $\mathrm{p} 38 \alpha$ and TAB1. A, In vitro interaction analysis between p38 $\alpha$ and TAB1. The profiles of size exclusion chromatography are shown for $\mathrm{p} 38 \alpha$ alone (blue), TAB1 alone (green) and their mixture (red). The corresponding SDS-PAGE results are shown on the right and boxed in respective color. $\mathrm{B}$, TAB1-induced autophosphorylaiton of $\mathrm{p} 38 \alpha$ on both $\mathrm{Thr}^{180}$ and $\mathrm{Tyr}^{182}$ in the activation loop. At indicated time intervals, aliquots of the reaction were removed and subjected to Western blot analysis using either anti- $p$ Thr or anti- $p$ Tyr antibodies. C, p38 $\alpha / p$ T $p \mathrm{Y}-\mathrm{catalyzed} \mathrm{TAB} 1$ phosphorylation. The phosphorylation rate of $\mathrm{p} 38 \alpha / p \mathrm{~T} p \mathrm{Y}$ towards its classical substrate ATF2 is shown for comparison. The reaction mixture contains 1 $\mathrm{nmol} \mathrm{L} \mathrm{L}^{-1}$ of $\mathrm{p} 38 \alpha / p \mathrm{~T} p \mathrm{Y}$ and $5 \mu \mathrm{mol} \mathrm{L} \mathrm{L}^{-1}$ of TAB1 or ATF2. 
might induce a conformational change of TAB1, impair the TAB1-TAK1 interaction and ultimately downregulate the TAB1-mediated TAK1 activation. Such a feedback inhibition is key to the intricate regulation of the TAK1-p38 $\alpha$ signaling [24-26].

\subsection{Overall structure of p38 $\alpha$-pepTAB1 complex}

Many MAPK interacting proteins have a conserved kinase interaction motif (KIM) or docking motif (D motif) with the consensus sequence of $(\mathrm{R} / \mathrm{K})_{1 \text { to } 2^{-}}(\mathrm{X})_{2-6}-\Phi_{\mathrm{A}}-\mathrm{X}-\Phi_{\mathrm{B}}$, where $\Phi_{\mathrm{A}}$ and $\Phi_{\mathrm{B}}$ represent the hydrophobic residues [15]. Zhou et al. [27] previously showed that TAB1 also contains a KIM sequence (residues 395-414) that plays a major role in high-affinity $\mathrm{p} 38 \alpha$ binding. We thus carried out crystallization trials of $\mathrm{p} 38 \alpha$ in complex with TAB1, but all efforts failed. We then generated fusion proteins in which a 21-residue peptide of TAB1 (residues 395-415, pepTAB1) was fused to the $\mathrm{C}$-terminus of $\mathrm{p} 38 \alpha$. This $\mathrm{p} 38 \alpha$-pepTAB1 complex structure was determined to $2.7 \AA$ resolution, and the statistics for data collection and structure refinement are summarized in Table 1.

In the complex, p38 $\alpha$ adopts the classical bilobal kinase fold, consisting of a small $\beta$-stranded $\mathrm{N}$-lobe and a larger $\alpha$-helical $\mathrm{C}$-lobe (Figure 2A). The TAB1 peptide

Table 1 Data collection and refinement statistics

\begin{tabular}{|c|c|}
\hline Data collection $^{\text {a) }}$ & p38 $\alpha$-pepTAB1 \\
\hline Space group & $P 3_{1} 21$ \\
\hline \multicolumn{2}{|l|}{ Cell dimensions } \\
\hline$a, b, c(\AA)$ & $82.1,82.1,122.6$ \\
\hline$\alpha, \beta, \gamma\left(^{\circ}\right)$ & $90,90,120$ \\
\hline Wavelength (̊) & 0.979413 \\
\hline Resolution $(\AA)$ & $50.0-2.7(2.76-2.71)^{\mathrm{b})}$ \\
\hline Completeness (\%) & $96.1(100.0)^{\mathrm{a})}$ \\
\hline Average $I / \sigma I$ & $21.1(4.9)$ \\
\hline Redundancy & $5.5(6.0)$ \\
\hline$R_{\text {merge }}$ & $0.061(0.457)$ \\
\hline Total number of observations & 71433 \\
\hline Total number unique & 13030 \\
\hline \multicolumn{2}{|l|}{ Refinement } \\
\hline Resolution $(\AA)$ & 2.7 \\
\hline Number of reflections & 13001 \\
\hline$R_{\text {work }} / R_{\text {free }}(\%)$ & $22.5 / 26.7$ \\
\hline \multicolumn{2}{|l|}{ Number of atoms/residues } \\
\hline Protein & $2799 / 348$ \\
\hline Water & 8 \\
\hline \multicolumn{2}{|l|}{ B-factor $\left(\AA^{2}\right)$} \\
\hline Protein & 70.4 \\
\hline Water & 45.0 \\
\hline Rmsd in bond length $(\AA)$ & 0.010 \\
\hline Rmsd in bond angles $\left({ }^{\circ}\right)$ & 1.241 \\
\hline \multicolumn{2}{|l|}{ Ramachandran-plot statistics } \\
\hline Favored regions (\%) & 91.6 \\
\hline Allowed regions (\%) & 8.4 \\
\hline Disallowed regions (\%) & 0 \\
\hline
\end{tabular}

a) Each data set was collected from a single crystal. b) Values in parentheses are for the highest resolution shell. $\left({ }^{406}\right.$ TLSLVMPS $\left.^{413}\right)$ was clearly visible and binds from the back of the active site, to a groove on the kinase $\mathrm{C}$-lobe (Figure 2B and C). The final model contains residues 4-354 of $\mathrm{p} 38 \alpha$ and residues $406-413$ from the TAB 1 peptide, and the extreme C-terminal 6 amino acids of p38 $\alpha$ and the adjacent $\mathrm{N}$-terminal 11 amino acids of TAB1 peptide were untraceable. In our crystallization experiments, the TAB1 peptide was fused to $\mathrm{p} 38 \alpha$. One potential caveat of the fusion approach is that the structure may deviate from that of the natural complex due to constraint in the movement of two fused proteins/peptides. It is important to note that the $\mathrm{C} \alpha$ distance between the C-terminal Asp354 of p38 $\alpha$ and the N-terminal Thr406 of the TAB1 peptide is approximately $22 \AA$ (Figure $2 \mathrm{~B}$ ), which would be readily covered by only seven residues. Thus, the 17 disordered residues of the p38 $\alpha$-pepTAB1 fusion protein probably form a flexible and long enough linker. We believe that the conformation of p38 $\alpha$-pepTAB1 complex is unlikely an "artifact" of the fusion protein.

\subsection{The KIM docking site on p38a}

There are two major elements found in nearly all KIMs: a cluster of basic residues (Arg or Lys) at the $\mathrm{N}$ terminus and a hydrophobic motif $\left(\Phi_{\mathrm{A}}-\mathrm{X}-\Phi_{\mathrm{B}}\right)$ near the $\mathrm{C}$ terminus [28]. The TAB1-KIM peptide does contain one basic residue Lys402, however, this residue is disordered in our structure. This observation suggests that the $\mathrm{CD}$ site for basic residue(s) may contribute little to the p38 $\alpha$-TAB1 interactions. On the other hand, the TAB1 peptide forms tight hydrophobic interactions through multiple hydrophobic side chains with the conserved hydrophobic docking groove of p38a, which is composed of helix $\alpha \mathrm{D}, \alpha \mathrm{E}$ and the reverse turn between $\beta 7$ and $\beta 8$ in p38 $\mathrm{C}$-lobe (Figures $2 \mathrm{~A}$ and $3 \mathrm{~A}$ ). Previous sequence analysis had suggested that the hydrophobic residues Leu407, Leu409 within the predicted $\Phi_{\mathrm{A}}-\mathrm{X}-\Phi_{\mathrm{B}}$ motif and an additional hydrophobic residue Pro412 are significant for TAB1 interaction with p38 $\alpha$ [27]. However, in our complex structure, the p38 $\alpha$ docking site for the $\Phi_{\mathrm{A}}-\mathrm{X}-\Phi_{\mathrm{B}}$ motif is bound by the ${ }^{409} \mathrm{LVM}^{411}$ segment of $\mathrm{TAB} 1$, inconsistent with previous prediction. Leu409 nestles into the $\Phi_{\mathrm{A}}$ binding pocket constituted by the hydrophobic side chains of Ile116, Leu122, His126, Val158 and Cys162, while Met411 makes multiple van der Waals contacts with the $\Phi_{\mathrm{B}}$ site formed by Ala111, Ile116 and Glu160 (Figure 3B). In addition to these conventional hydrophobic interactions, Pro412 at the $\Phi_{\mathrm{B}}+1$ site of TAB1-KIM is surrounded by the side chains of Ala111, Ile116, Asn115 and Cys119, and Leu407 at the $\Phi_{\mathrm{A}}-2$ site penetrates into a hydrophobic pocket formed by His126, Phe129, Cys162 and Tyr311 (Figure 3C). Furthermore, the main chain amide and carbonyl groups of the TAB1 ${ }^{408} \mathrm{SLV}^{410}$ segment are hydrogen bonded by the side chains of His126, Asn120 and the main chain of Glu160 from p38 $\alpha$, which further stabilize the interaction between $\mathrm{p} 38 \alpha$ and 

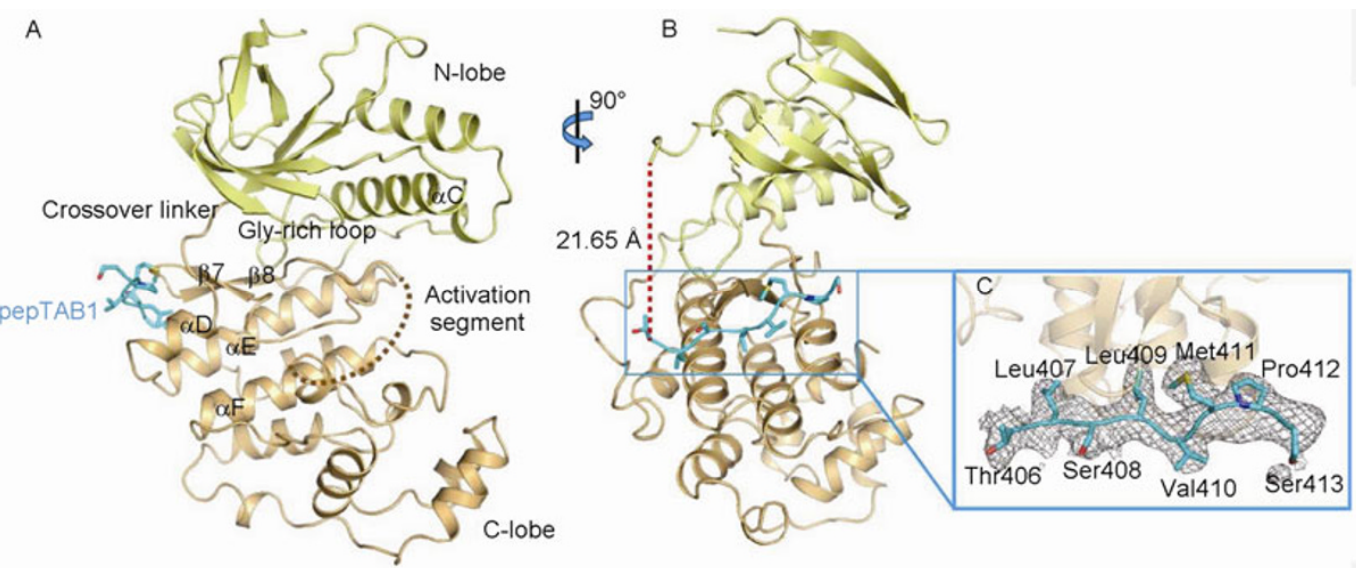

Figure 2 Structure of the p38 $\alpha$-pepTAB1 complex. A and B, Schematic diagram of the p38 $\alpha$-pepTAB1 structure in two views related by a $90^{\circ}$ rotation around a vertical axis. The color scheme is $\mathrm{p} 38 \alpha$ (N lobe, pale yellow; $\mathrm{C}$ lobe, light orange) and pepTAB1 (cyan). The dotted line in panel A indicates the disordered middle portion of $\mathrm{p} 38 \alpha$ activation segment, and that in panel B indicates the distance between the traceable p38 $\alpha$ C-terminus and pepTAB1 N-terminus. C, SA omit map (contoured at $1.5 \sigma$, grey mesh) for the traceable pepTAB1 (residues 406-413).

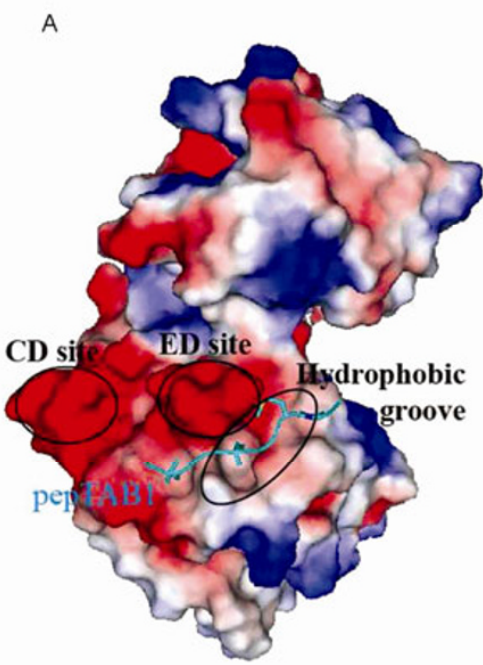

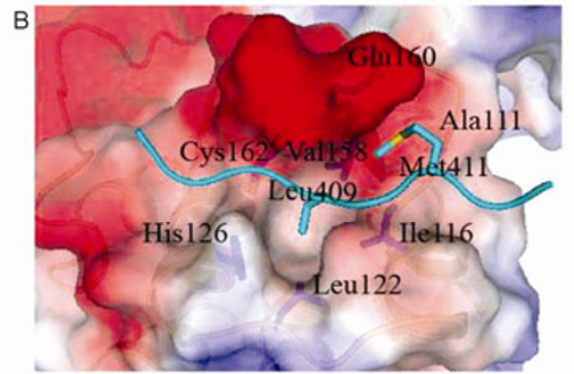

D

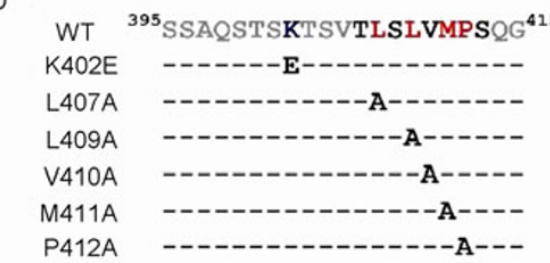

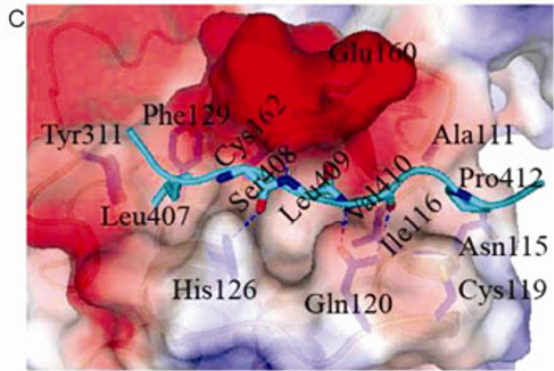

Interaction with $\mathrm{p} 38 \alpha$

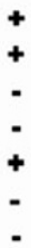

Figure 3 Interaction between $\mathrm{p} 38 \alpha$ and pepTAB1. A, Surface representation of the p38 $\alpha$-pepTAB1 complex. The p38 $\alpha$ is colored according to electrostatic potential (positive, blue; negative, red), and pepTAB1 is shown in cyan ribbon/sticks. The classical CD and ED sites on p38 $\alpha$ for basic residue binding and the hydrophobic groove accommodating the $\Phi_{\mathrm{A}}$ and $\Phi_{\mathrm{B}}$ side chains of KIMs were indicated. B, Close-up view of the classical hydrophobic binding pockets on p38 $\alpha$ for pepTAB1 residues Leu409 $\left(\Phi_{\mathrm{A}}\right)$ and Met411 $\left(\Phi_{\mathrm{B}}\right)$. C, Close-up view of two additional hydrophobic binding pockets on p38 $\alpha$ for pepTAB1 residues Leu407 $\left(\Phi_{\mathrm{A}}-2\right)$ and Pro412 $\left(\Phi_{\mathrm{B}}+1\right)$. The hydrogen bonds formed between p38 $\alpha$ and pepTAB1 were indicated by blue dashed lines. D, Interactions of TAB1 mutants with $\mathrm{p} 38 \alpha$. The basic and hydrophobic residues on TAB1-KIM peptide were mutated individually, and the p38 $\alpha$ interaction of these TAB1 mutants were examined via size exclusion chromatography.

TAB1 peptide (Figure 3C). Therefore, the TAB1-KIM peptide binds to the canonical groove of $\mathrm{p} 38 \alpha$, yet in a similar mode from those observed in other p38a-KIM complex structures $[29,30]$. To assess the importance of the observed interactions, we carried out site directed mutagenesis analyses on the KIM of TAB1 and examined their effects on the p38 $\alpha$-TAB1 interaction by gel filtration. The results correlate well with the structure analysis (Figure 3D).

\subsection{Long-range conformational changes of p38a}

To better understand the role of TAB1 peptide binding, we compared the p38 $\alpha$-pepTAB1 complex structure with those of the inactive unphosphorylated p38 $\alpha$ (PDB ID: 1P38) and the active dual-phosphorylated p38 $\alpha$ (PDB ID: 3PY3) [31-33]. The binding of TAB1 peptide induces long-range conformational changes of p38 $\alpha$ (Figure 4). In the reported p38 $\alpha$ structures, the activation segments between and including the important DFG and APE motifs are both well-ordered, though adopting distinct conformations (Figure 4A). However, the middle segment of the activation segment (residues 173-183) is disordered in our complex structure, while the other traceable residues exhibit the same conformation as that in the unphosphorylated $\mathrm{p} 38 \alpha$. 

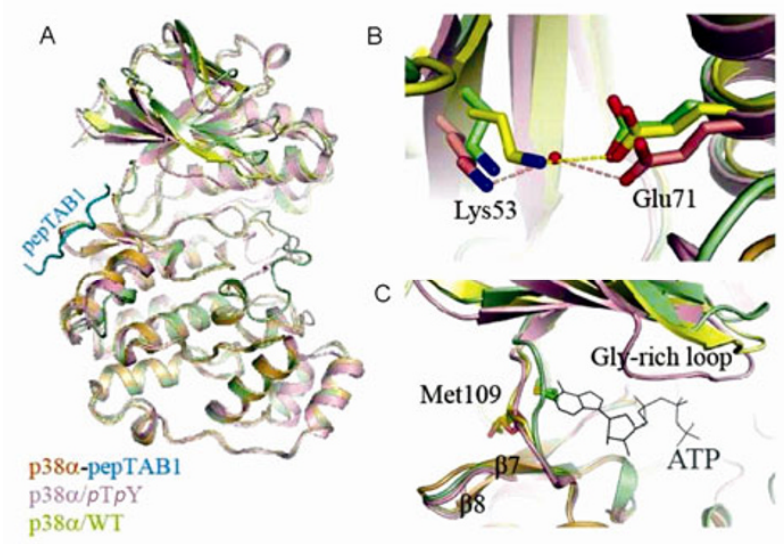

Figure 4 TAB1-induced conformational changes of p38 $\alpha$. A, Superposition of the p38 $\alpha$-pepTAB1 complex (colored as that in Figure 2A) with wildtype $\mathrm{p} 38 \alpha$ (PDB ID: 1P38, pale green) and active $\mathrm{p} 38 / p \mathrm{~T} p \mathrm{Y}$ (PDB ID: 3PY3, light pink). B, Close-up view of the salt bridge(s) formed between Lys53 and Glu71. The side chains are highlighted as sticks, and the water molecule is shown as red dot. C, Close-up view of the ATP binding pocket. The ATP molecule is adapted from the structure of $\mathrm{p} 38 \gamma / p \mathrm{~T} p \mathrm{Y}$ (PDB ID: 1CM8) and shown as black lines.

One hallmark of protein kinases in their activated state is the formation of salt bridge(s) between a Lys on strand $\beta 3$ and a Glu from helix $\alpha \mathrm{C}$, where the Lys can interact with the $\alpha$ - and $\beta$-phosphates of a bound ATP molecule [34-36]. No salt bridge was observed between the highly conserved Lys53 and Glu71 in the unphosphorylated p38 , by contrast, a water-mediated hydrogen bond was formed in the dual-phosphorylated p38 $\alpha$. Interestingly, a direct salt bridge between Lys53 and Glu71 is observed in our p38 $\alpha$ pepTAB1 complex structure (Figure 4B). Similar conformational changes occur in complexes between $\mathrm{p} 38 \alpha$ and other KIM-peptide, p38 $\alpha$-pepMKK3b (PDB ID: 1LEZ), p38 $\alpha$-pepMEF2A (PDB ID: 1LEW) [29].

Another notable change also occurs at the ATP binding pocket, a deep cleft between the $\mathrm{N}$ - and C-lobes of p38 $\alpha$ kinase (Figure 4C). The highly conserved Gly-rich loop lies on the top of a bound ATP molecule and plays an important role in stabilizing the phosphate groups of ATP [34-36]. The binding of TAB1 peptide to the inactive $\mathrm{p} 38 \alpha$ induces a slightly downward movement of the Gly-rich loop, which thus adopts a conformation in between those in the inactive and active p38 $\alpha$ states. The same is true for the other KIM-peptide bound p38 structures [29,35]. The crossover linker $\left({ }^{107} \mathrm{HLMGAD}^{112}\right)$ connecting the $\mathrm{N}$ - and C-lobes of p38 $\alpha$ constitutes one wall of the ATP-binding pocket, which also undergoes conformational change upon TAB1-KIM binding. In particular, the side chain of Met109 in the inactive p38 $\alpha$ points into the cleft and sterically crash with the adenine ring, while Met109 in the fully active p38 $\alpha$, as well as in our p38 $\alpha$-pepTAB1 complex, rotates away from the active site and occupies a new pocket formed by the $\beta 7-\beta 8$ hairpin (Figure 4C). Met109 in the latter cases allows the binding of ATP. Thus, the conformational change of the crossover connection, particularly that of Met109, may influence the binding affinity of ATP. Close inspection of the peptide bound structures indicate that Met411 $\left(\Phi_{\mathrm{B}}\right)$ and Pro412 $\left(\Phi_{\mathrm{B}}+1\right)$ form hydrophobic interactions with the residues within/near the crossover connection, which may affect the orientation of Met109. Therefore, the binding of TAB1-KIM peptide induces long-range conformational changes of the inactive $\mathrm{p} 38 \alpha$ towards the activated state.

\subsection{The R-spine and C-spine}

To further assess the KIM-induced conformational changes of $\mathrm{p} 38 \alpha$, we then investigated the catalytic spine (C-spine) and the regulatory spine (R-spine), two conserved structural and functional elements of protein kinases (Figure 5A). The integrities of these spines are important for the binding of ATP and substrate and thereby imply the active state of protein kinases [34]. The C-spine of p38 $\alpha$ is composed of eight highly conserved hydrophobic residues from both $\mathrm{N}$ and C-lobes, consisting of Val38, Ala51, Leu113, Leu156, Ala157, Val158, Ile212 and Leu216 (Figure 5B). In particular, the side chains of Val38, Ala51 from the N-Lobe and Ala157 from the C-lobe form a hydrophobic pocket for accommodating the adenine ring of a bound-ATP molecule. The p38 $\alpha$-pepTAB1 complex adopts an intermediate conformation different from either the inactive or active $\mathrm{p} 38 \alpha$ structures. The rest $\mathrm{C}$-spine residues are buried deeply in the C-lobe and change little upon KIM peptide binding or phosphorylation activation. The R-spine is formed by four non-consecutive hydrophobic residues, Leu75 from strand $\beta 4$, Leu86 from the universally conserved helix $\alpha \mathrm{C}$, His 148 on the catalytic loop and Phe169 in the conserved DFG motif (Figure 5B). Distinct from that in the active p38 $\alpha$ structure, Phe169 in our p38 $\alpha$-TAB1 complex and the inactive p38 $\alpha$ structure shifts slightly downwards to the C-lobe, which only interacts with His148 in the C-lobe, but not Leu75 from the N-lobe. In addition, the adjacent Asp168 in the DFG motif is inappropriately positioned and thus unable to ligate the potential ATP molecule (Figure 5B-D). Thus, the TAB1-bound p38 $\alpha$ does not adopt the fully active conformation, which corroborates the slow rate of TAB1induced $\mathrm{p} 38 \alpha$ autophosphorylation.

In summary, we solved the crystal structure of inactive p38 $\alpha$ in complex with the KIM peptide derived from TAB1. The structure reveals that the TAB1-KIM peptide binds to p38 $\alpha$ mainly via hydrophobic docking interactions, which triggers the inactive $\mathrm{p} 38 \alpha$ to undergo long-range conformational changes, particularly at the active site, towards the active kinase conformation. Notably, the TAB1-KIM bound p38 $\alpha$ structure yet exhibits significant differences from the full active p38 $\alpha$ conformation, suggesting that the TAB1-KIM peptide alone may be insufficient to trigger the full activation of $\mathrm{p} 38 \alpha$. Nevertheless, we believe that TAB1 is a reasonable substrate of $\mathrm{p} 38 \alpha$. In consideration of 


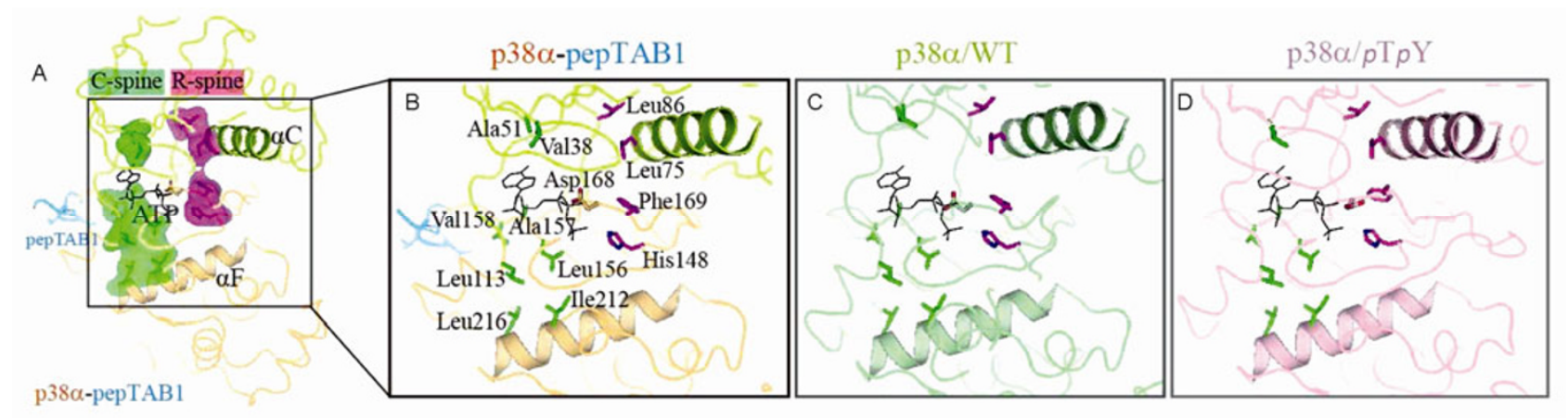

Figure 5 Comparison of the R-spine and C-spine of p38 $\alpha$-pepTAB1, p38 $\alpha /$ WT and $\mathrm{p} 38 \alpha / p \mathrm{~T} p \mathrm{Y}$. The color schemes for the three $\mathrm{p} 38 \alpha$ structures are the same as that in Figure 4A, and the helices $\alpha \mathrm{C}$ and $\alpha \mathrm{F}$ are highlighted as cartoon. A, Residues composing the R-spine and C-spine in the p38 $\alpha$-pepTAB1 complex are highlighted in green and magenta sticks and surfaces, respectively. B, Close-up view of the R-spine and C-spine in the p38 $\alpha$-pepTAB1 complex. $\mathrm{C}$, Close-up view of the $\mathrm{R}$-spine and $\mathrm{C}$-spine in the wildtype $\mathrm{p} 38 \alpha$ structure. $\mathrm{D}$, Close-up view of the $\mathrm{R}$-spine and $\mathrm{C}$-spine in the active $\mathrm{p} 38 \alpha / p \mathrm{~T} p \mathrm{Y}$ structure.

the different roles of TAB1 being a regulator of $\mathrm{p} 38 \alpha$ autophosphorylation and/or a downstream substrate of $\mathrm{p} 38 \alpha$, we aspire to get more information from structural studies of p38 $\alpha$ in complex with full-length TAB1.

Crystallographic data were collected at Shanghai Synchrotron Radiation Facility beamline 17U. This work was supported in part by National Natural Science Foundation of China $(31130062,31070643)$ and Tsinghua University (20121080028).

1 Tanno M, Bassi R, Gorog D A, et al. Diverse mechanisms of myocardial p38 mitogen-activated protein kinase activation: evidence for MKK-independent activation by a TAB1-associated mechanism contributing to injury during myocardial ischemia. Circ Res, 2003, 93: 254-261

2 Kyriakis J M, Avruch J. Mammalian mitogen-activated protein kinase signal transduction pathways activated by stress and inflammation. Physiol Rev, 2001, 81: 807-869

3 Qi M, Elion E A. MAP kinase pathways. J Cell Sci, 2005, 118: 3569-3572

4 Enslen H, Brancho D M, Davis R J. Molecular determinants that mediate selective activation of $\mathrm{p} 38$ MAP kinase isoforms. EMBO J, 2000, 19: 1301-1311

5 Ge B, Gram H, Di Padova F, et al. MAPKK-independent activation of p38alpha mediated by TAB1-dependent autophosphorylation of p38alpha. Science, 2002, 295: 1291-1294

6 Salvador J M, Mittelstadt P R, Guszczynski T, et al. Alternative p38 activation pathway mediated by $\mathrm{T}$ cell receptor-proximal tyrosine kinases. Nat Immunol, 2005, 6: 390-395

7 Tzarum N, Diskin R, Engelberg D, et al. Active mutants of the TCR-mediated p38alpha alternative activation site show changes in the phosphorylation lip and DEF site formation. J Mol Biol, 2011, 405: 1154-1169

8 Gills J J, Castillo S S, Zhang C, et al. Phosphatidylinositol ether lipid analogues that inhibit AKT also independently activate the stress kinase, p38alpha, through MKK3/6-independent and -dependent mechanisms. J Biol Chem, 2007, 282: 27020-27029

9 Tzarum N, Eisenberg-Domovich Y, Gills J J, et al. Lipid molecules induce p38alpha activation via a novel molecular switch. J Mol Biol, 2012, 424: 339-353

10 Hanafusa $\mathrm{H}$, Ninomiya-Tsuji J, Masuyama N, et al. Involvement of the p38 mitogen-activated protein kinase pathway in transforming growth factor-beta-induced gene expression. J Biol Chem, 1999, 274: 27161-27167

11 Yu Y, Ge N, Xie M, et al. Phosphorylation of Thr-178 and Thr-184 in the TAK1 T-loop is required for interleukin (IL)-1-mediated optimal NFkappaB and AP-1 activation as well as IL-6 gene expression. J Biol Chem, 2008, 283: 24497-24505

12 Singhirunnusorn P, Suzuki S, Kawasaki N, et al. Critical roles of threonine 187 phosphorylation in cellular stress-induced rapid and transient activation of transforming growth factor-beta-activated kinase 1 (TAK1) in a signaling complex containing TAK1-binding protein TAB1 and TAB2. J Biol Chem, 2005, 280: 7359-7368

13 Kishimoto K, Matsumoto K, Ninomiya-Tsuji J. TAK1 mitogenactivated protein kinase kinase kinase is activated by autophosphorylation within its activation loop. J Biol Chem, 2000, 275: 7359-7364

14 Kumphune S, Chattipakorn S, Chattipakorn N. Role of $\mathrm{p} 38$ inhibition in cardiac ischemia/reperfusion injury. Eur J Clin Pharmacol, 2012, 68: 513-524

15 Denise Martin E, De Nicola G F, Marber M S. New therapeutic targets in cardiology: p38 alpha mitogen-activated protein kinase for ischemic heart disease. Circulation, 2012, 126: 357-368

16 Ohkusu-Tsukada K, Tominaga N, Udono H, et al. Regulation of the maintenance of peripheral T-cell anergy by TAB1-mediated p38 alpha activation. Mol Cell Biol, 2004, 24: 6957-6966

17 Ota A, Zhang J, Ping P, et al. Specific regulation of noncanonical p38alpha activation by Hsp90-Cdc37 chaperone complex in cardiomyocyte. Circ Res, 2010, 106: 1404-1412

18 Cheung P C, Campbell D G, Nebreda A R, et al. Feedback control of the protein kinase TAK1 by SAPK2a/p38alpha. EMBO J, 2003, 22: 5793-5805

19 Otwinowski Z, Minor W. Processing of X-ray diffraction data collected in oscillation mode. Macromol Crystallogr Part A, 1997, 276: 307-326

20 McCoy A J, Grosse-Kunstleve R W, Storoni L C, et al. Likelihood-enhanced fast translation functions. Acta Crystallogr D Biol Crystallogr, 2005, 61: 458-464

21 Adams P D, Grosse-Kunstleve R W, Hung L W, et al. PHENIX: building new software for automated crystallographic structure determination. Acta Crystallogr D Biol Crystallogr, 2002, 58: 1948-1954

22 Emsley P, Cowtan K. Coot: model-building tools for molecular graphics. Acta Crystallogr D Biol Crystallogr, 2004, 60: 2126-2132

23 Laskowski R A, Macarthur M W, Moss D S, et al. Procheck-a program to check the stereochemical quality of protein structures. J Appl Crystallogr, 1993, 26: 283-291

24 Gaestel M, Kotlyarov A, Kracht M. Targeting innate immunity protein kinase signalling in inflammation. Nat Rev Drug Discov, 2009, 8: 480-499

25 Cuadrado A, Nebreda A R. Mechanisms and functions of p38 MAPK signalling. Biochem J, 2010, 429: 403-417

26 Sakurai H. Targeting of TAK1 in inflammatory disorders and cancer. Trends Pharmacol Sci, 2012, 33: 522-530 
27 Zhou H, Zheng M, Chen J, et al. Determinants that control the specific interactions between TAB1 and p38alpha. Mol Cell Biol, 2006, 26: 3824-3834

28 Goldsmith E J, Akella R, Min X, et al. Substrate and docking interactions in serine/threonine protein kinases. Chem Rev, 2007, 107: 5065-5081

29 Chang C I, Xu B E, Akella R, et al. Crystal structures of MAP kinase p38 complexed to the docking sites on its nuclear substrate MEF2A and activator MKK3b. Mol Cell, 2002, 9: 1241-1249

30 Garai A, Zeke A, Gogl G, et al. Specificity of linear motifs that bind to a common mitogen-activated protein kinase docking groove. Sci Signal, 2012, 5: ra74

31 Wilson K P, Fitzgibbon M J, Caron P R, et al. Crystal structure of p38 mitogen-activated protein kinase. J Biol Chem, 1996, 271:
27696-27700

32 Wang Z, Harkins P C, Ulevitch R J, et al. The structure of mitogen-activated protein kinase p38 at 2.1-A resolution. Proc Natl Acad Sci USA, 1997, 94: 2327-2332

33 Zhang Y Y, Wu J W, Wang Z X. Mitogen-activated protein kinase (MAPK) phosphatase 3-mediated cross-talk between MAPKs ERK2 and p38alpha. J Biol Chem, 2011, 286: 16150-16162

34 Taylor S S, Kornev A P. Protein kinases: evolution of dynamic regulatory proteins. Trends Biochem Sci, 2011, 36: 65-77

35 Huse M, Kuriyan J. The conformational plasticity of protein kinases. Cell, 2002, 109: 275-282

36 Nolen B, Taylor S, Ghosh G. Regulation of protein kinases: controlling activity through activation segment conformation. Mol Cell, 2004, 15: 661-675

Open Access This article is distributed under the terms of the Creative Commons Attribution License which permits any use, distribution, and reproduction in any medium, provided the original author(s) and source are credited. 